\title{
The Impact of Exploiting Instruction-Level Parallelism on Shared-Memory Multiprocessors
}

\author{
Vijay S. Pai, Student Member, IEEE, Parthasarathy Ranganathan, Student Member, IEEE, \\ Hazim Abdel-Shafi, Student Member, IEEE, and Sarita Adve, Member, IEEE
}

\begin{abstract}
Current microprocessors incorporate techniques to aggressively exploit instruction-level parallelism (ILP). This paper evaluates the impact of such processors on the performance of shared-memory multiprocessors, both without and with the latencyhiding optimization of software prefetching. Our results show that, while ILP techniques substantially reduce CPU time in multiprocessors, they are less effective in removing memory stall time. Consequently, despite the inherent latency tolerance features of ILP processors, we find memory system performance to be a larger bottleneck and parallel efficiencies to be generally poorer in ILPbased multiprocessors than in previous generation multiprocessors. The main reasons for these deficiencies are insufficient opportunities in the applications to overlap multiple load misses and increased contention for resources in the system. We also find that software prefetching does not change the memory bound nature of most of our applications on our ILP multiprocessor, mainly due to a large number of late prefetches and resource contention. Our results suggest the need for additional latency hiding or reducing techniques for ILP systems, such as software clustering of load misses and producer-initiated communication.
\end{abstract}

Index Terms-Shared-memory multiprocessors, instruction-level parallelism, software prefetching, performance evaluation.

\section{INTRODUCTION}

$S^{\prime}$ HARED-MEMORY multiprocessors built from commodity microprocessors are being increasingly used to provide high performance for a variety of scientific and commercial applications. Current commodity microprocessors improve performance by using aggressive techniques to exploit high levels of instruction-level parallelism (ILP). These techniques include multiple instruction issue, out-of-order (dynamic) scheduling, nonblocking loads, and speculative execution. We refer to these techniques collectively as ILP techniques and to processors that exploit these techniques as ILP processors. Most previous studies of shared-memory multiprocessors, however, have assumed a simple processor with single-issue, in-order scheduling, blocking loads, and no speculation. A few multiprocessor architecture studies model state-of-the-art ILP processors [2], [7], [8], [9], but do not analyze the impact of ILP techniques.

To fully exploit recent advances in uniprocessor technology for shared-memory multiprocessors, a detailed analysis of how ILP techniques affect the performance of such systems and how they interact with previous optimizations for such systems is required. This paper evaluates the impact of exploiting ILP on the performance of sharedmemory multiprocessors, both without and with the latency-hiding optimization of software prefetching. ${ }^{1}$

For our evaluations, we study five applications using detailed simulation, described in Section 2.

Section 3 analyzes the impact of ILP techniques on the performance of shared-memory multiprocessors without the use of software prefetching. All our applications see

- The authors are with the Department of Electrical and Computer Engineering, Rice University, Houston, TX 77251-1892.

E-mail: \{vijaypai, parthas, shafi, sarita\}@rice.edu.

For information on obtaining reprints of this article, please send e-mail to: tc@computer.org, and reference IEEECS Log Number 108235. performance improvements from the use of current ILP techniques, but the improvements vary widely. In particular, ILP techniques successfully and consistently reduce the CPU component of execution time, but their impact on the memory stall time is lower and more applicationdependent. Consequently, despite the inherent latency tolerance features integrated within ILP processors, we find memory system performance to be a larger bottleneck and parallel efficiencies to be generally poorer in ILP-based multiprocessors than in previous-generation multiprocessors. These deficiencies are caused by insufficient opportunities in the application to overlap multiple load misses and increased contention for system resources from more frequent memory accesses.

Software-controlled nonbinding prefetching has been shown to be an effective technique for hiding memory latency in simple processor-based shared memory systems [6]. Section 4 analyzes the interaction between software prefetching and ILP techniques in shared-memory multiprocessors. We find that, compared to previous generation systems, increased late prefetches and increased contention for resources cause software prefetching to be less effective in reducing memory stall time in ILP-based systems. Thus, even after adding software prefetching, most of our applications remain largely memory bound on the ILPbased system.

Overall, our results suggest that, compared to previousgeneration shared-memory systems, ILP-based systems have a greater need for additional techniques to tolerate or reduce memory latency. Specific techniques motivated

1. This paper combines results from two previous conference papers [11], [12], using a common set of system parameters, a more aggressive MESI (vs. MSI) cache-coherence protocol, a more aggressive compiler (the better of SPARC SC 4.2 and gcc 2.7.2 for each application, rather than gcc 2.5.8), and full simulation of private memory references. 


\begin{tabular}{|l|l|}
\hline \multicolumn{2}{|c|}{ Processor parameters } \\
\hline Clock rate & $300 \mathrm{MHz}$ \\
$\begin{array}{l}\text { Fetch/decode/retire rate } \\
\text { Instruction window (re- } \\
\text { order buffer) size }\end{array}$ & 4 per cycle \\
$\begin{array}{l}\text { Memory queue size } \\
\text { Outstanding branches }\end{array}$ & 32 \\
Functional units & 8 \\
\hline \multicolumn{2}{|c|}{ Memory hierarchy and network parameters } \\
\hline L1 cache & $16 \mathrm{~KB}$, direct-mapped, 2 ports, 8 MSHRs, 64-byte line \\
L2 cache & $64 \mathrm{~KB}, 4$-way associative, 1 port, 8 MSHRs, 64-byte line, pipelined \\
Memory & 4 -way interleaved, 60 ns access time, 16 bytes/cycle \\
Bus & $100 \mathrm{MHz}, 128$ bits, split transaction \\
Network & $2 \mathrm{D}$ mesh, 150MHz, 64 bits; per hop flit delay of 2 network cycles \\
Nodes in multiprocessor & 8 \\
\hline \multicolumn{2}{|c|}{ Resulting contentionless latencies (in processor cycles) } \\
\hline L1 hit & 1 cycle \\
L2 hit & 10 cycles \\
Local memory & 45 cycles \\
Remote memory & $140-220$ cycles \\
Cache-to-cache transfer & $170-270$ cycles \\
\hline
\end{tabular}

Fig. 1. System parameters.

by our results include clustering of load misses in the applications to increase opportunities for load misses to overlap with each other and techniques such as producerinitiated communication that reduce latency to make prefetching more effective (Section 5).

\section{Methodology}

\subsection{Simulated Architectures}

To determine the impact of ILP techniques on multiprocessor performance, we compare two systems-ILP and Simple-equivalent in every respect except the processor used. The ILP system uses state-of-the-art ILP processors while the Simple system uses simple processors (Section 2.1.1). We compare the ILP and Simple systems not to suggest any architectural trade-offs but, rather, to understand how aggressive ILP techniques impact multiprocessor performance. Therefore, the two systems have identical clock rates and include identical aggressive memory and network configurations suitable for the ILP system (Section 2.1.2). Fig. 1 summarizes all the system parameters.

\subsubsection{Processor Models}

The ILP system uses state-of-the-art processors that include multiple issue, out-of-order (dynamic) scheduling, nonblocking loads, and speculative execution. The Simple system uses previous-generation simple processors with single issue, in-order (static) scheduling, and blocking loads, and represents commonly studied shared-memory systems. Since we did not have access to a compiler that schedules instructions for our in-order simple processor, we assume single-cycle functional unit latencies (as also assumed by most previous simple-processor based shared-memory studies). Both processor models include support for software-controlled nonbinding prefetching to the L1 cache.

\subsubsection{Memory Hierarchy and Multiprocessor Configuration}

We simulate a hardware cache-coherent, nonuniform memory access (CC-NUMA) shared-memory multiprocessor using an invalidation-based, four-state MESI directory coherence protocol [4]. We model release consistency because previous studies have shown that it achieves the best performance [9].

The processing nodes are connected using a twodimensional mesh network. Each node includes a processor, two levels of caches, a portion of the global sharedmemory and directory, and a network interface. A splittransaction bus connects the network interface, directory controller, and the rest of the system node. Both caches use a write-allocate, write-back policy. The cache sizes are chosen commensurate with the input sizes of our applications, following the methodology described by Woo et al. [14]. The primary working sets for our applications fit in the L1 cache, while the secondary working sets do not fit in the L2 cache. Both caches are nonblocking and use miss status holding registers (MSHRs) [3] to store information on outstanding misses and to coalesce multiple requests to the same cache line. All multiprocessor results reported in this paper use a configuration with eight nodes. 


\subsection{Simulation Environment}

We use RSIM, the Rice Simulator for ILP Multiprocessors, to model the systems studied [10]. RSIM is an executiondriven simulator that models the processor pipelines, memory system, and interconnection network in detail, including contention at all resources. It takes SPARC application executables as input. To speed up our simulations, we assume that all instructions hit in the instruction cache. This assumption is reasonable since all our applications have very small instruction footprints.

\subsection{Performance Metrics}

In addition to comparing execution times, we also report the individual components of execution time-CPU, data memory stall, and synchronization stall times-to characterize the performance bottlenecks in our systems. With ILP processors, it is unclear how to assign stall time to specific instructions since each instruction's execution may be overlapped with both preceding and following instructions. We use the following convention, similar to previous work (e.g., [5]), to account for stall cycles. At every cycle, we calculate the ratio of the instructions retired from the instruction window in that cycle to the maximum retire rate of the processor and attribute this fraction of the cycle to the busy time. The remaining fraction of the cycle is attributed as stall time to the first instruction that could not be retired that cycle. We group the busy time and functional unit (nonmemory) stall time together as CPU time. Henceforth, we use the term memory stall time to denote the data memory stall component of execution time.

In the first part of the study, the key metric used to evaluate the impact of ILP is the ratio of the execution time with the Simple system relative to that achieved by the ILP system, which we call the ILP speedup. For detailed analysis, we analogously define an ILP speedup for each component of execution time.

\subsection{Applications}

Fig. 2 lists the applications and the input sets used in this study. Radix, LU, and FFT are from the SPLASH-2 suite [14], and Water and Mp3d are from the SPLASH suite [13]. These five applications and their input sizes were chosen to ensure reasonable simulation times. (Since RSIM models aggressive ILP processors in detail, it is about 10 times slower than simple-processor-based shared-memory simulators.) LUopt and FFTopt are versions of LU and FFT that include ILP-specific optimizations that can potentially be implemented in a compiler. Specifically, we use function inlining and loop interchange to move load misses closer to each other so that they can be overlapped in the ILP processor. The impact of these optimizations is discussed in Sections 3 and 5. Both versions of LU are also modified slightly to use flags instead of barriers for better load balance.

Since a SPARC compiler for our ILP system does not exist, we compiled our applications with the commercial Sun SC 4.2 or the gcc 2.7.2 compiler (based on better simulated ILP system performance) with full optimization turned on. The compilers' deficiencies in addressing the specific instruction grouping rules of our ILP system are partly hidden by the out-of-order scheduling in the ILP processor. $^{2}$

\section{Impact of ILP Techniques on Performance}

This section analyzes the impact of ILP techniques on multiprocessor performance by comparing the simple and ILP systems, without software prefetching.

\subsection{Overall Results}

Figs. 3 and 4 illustrate our key overall results. For each application, Fig. 3 shows the total execution time and its three components for the Simple and ILP systems (normalized to the total time on the simple system). Additionally, at the bottom, the figure also shows the ILP speedup for each application. Fig. 4 shows the parallel efficiency $^{3}$ of the ILP and Simple systems expressed as a percentage. These figures show three key trends:

- ILP techniques improve the execution time of all our applications. However, the ILP speedup shows a wide variation (from 1.29 in Mp3d to 3.54 in LUopt). The average ILP speedup for the original applications (i.e., not including LUopt and FFTopt) is only 2.05.

- The memory stall component is generally a larger part of the overall execution time in the ILP system than in the Simple system.

- Parallel efficiency for the ILP system is less than that for the Simple system for all applications.

We next investigate the reasons for the above trends.

\subsection{Factors Contributing to ILP Speedup}

Fig. 3 indicates that the most important components of execution time are CPU time and memory stalls. Thus, ILP speedup will be shaped primarily by CPU ILP speedup and memory ILP speedup. Fig. 5 summarizes these speedups (along with the total ILP speedup). The figure shows that the low and variable ILP speedup for our applications can be attributed largely to insufficient and variable memory ILP speedup; the CPU ILP speedup is similar and significant among all applications (ranging from 2.94 to 3.80). More detailed data shows that, for most of our applications, memory stall time is dominated by stalls due to loads that miss in the L1 cache. We therefore focus on the impact of ILP on (L1) load misses below.

The load miss ILP speedup is the ratio of the stall time due to load misses in the Simple and ILP systems and is determined by three factors, described below. The first factor increases the speedup, the second decreases it, while the third may either increase or decrease it.

- Load miss overlap. Since the Simple system has blocking loads, the entire load miss latency is exposed as stall time. In ILP, load misses can be overlapped with other useful work, reducing stall time and increasing the ILP load miss speedup. The

2. To the best of our knowledge, the key compiler optimization identified in this paper (clustering of load misses) is not implemented in any current superscalar compiler.

3. The parallel efficiency for an application on a system with $N$ processors is defined as $\frac{\text { Execution time on uniprocessor }}{\text { Execution time on multiprocessor }} \times \frac{1}{N}$ 


\begin{tabular}{|l|l|}
\hline Application & \multicolumn{1}{|c|}{ Input Size } \\
\hline LU, LUopt & 256x256 matrix, block 8 \\
FFT, FFTopt & 65536 points \\
Mp3d & 50000 particles \\
Water & 512 molecules \\
Radix & 1024 radix, 512K keys, max 512K \\
\hline
\end{tabular}

Fig. 2. Applications and input sizes.

number of instructions behind which a load miss can overlap is, however, limited by the instruction window size; further, load misses have longer latencies than other instructions in the instruction window. Therefore, load miss latency can normally be completely hidden only behind other load misses. Thus, for significant load miss ILP speedup, applications should have multiple load misses clustered together within the instruction window to enable these load misses to overlap with each other.

- Contention. Compared to the Simple system, the ILP system can see longer latencies from increased contention due to the higher frequency of misses, thereby negatively affecting load miss ILP speedup.

- Change in the number of misses. The ILP system may see fewer or more misses than the Simple system because of speculation or reordering of memory accesses, thereby positively or negatively affecting load miss ILP speedup.

All of our applications except LU see a similar number of cache misses in both the Simple and ILP case. LU sees 2.5 times fewer misses in ILP because of a reordering of accesses that otherwise conflict. When the number of misses does not change, the ILP system sees $(>1)$ load miss ILP speedup if the load miss overlap exploited by ILP outweighs any additional latency from contention. We illustrate the effects of load miss overlap and contention using the two applications that best characterize them: LUopt and Radix.

Fig. 6a provides the average load miss latencies for LUopt and Radix in the Simple and ILP systems, normalized to the Simple system latency. The latency shown is the total miss latency, measured from address generation to data arrival, including the overlapped part (in ILP) and the exposed part that contributes to stall time. The difference in

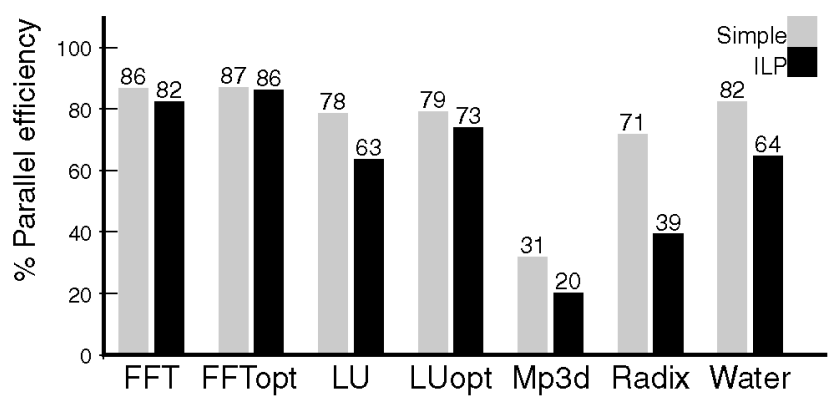

Fig. 4. Impact of ILP on parallel efficiency.

the bar lengths of Simple and ILP indicates the additional latency added due to contention in ILP. Both of these applications see a significant latency increase from resource contention in ILP. However, LUopt can overlap all its additional latency, as well as a large portion of the base (Simple) latency, thus leading to a high memory ILP speedup. On the other hand, Radix cannot overlap its additional latency; thus, it sees a load miss slowdown in the ILP configuration.

We use the data in Figs. $6 \mathrm{~b}$ and $6 \mathrm{c}$ to further investigate the causes for the load miss overlap and contention-related latencies in these applications.

\subsubsection{Causes for Load Miss Overlap}

Fig. $6 \mathrm{~b}$ shows the ILP system's L1 MSHR occupancy due to load misses for LUopt and Radix. Each curve shows the fraction of total time for which at least $\mathrm{N}$ MSHRs are occupied by load misses, for each possible $\mathrm{N}$ (on the $\mathrm{X}$ axis). This figure shows that LUopt achieves significant overlap of load misses, with up to eight load miss requests outstanding simultaneously at various times. In contrast, Radix almost never has more than one outstanding load miss at any time. This difference arises because load misses are clustered together in the instruction window in LUopt, but typically separated by too many instructions in Radix.

\subsubsection{Causes for Contention}

Fig. $6 \mathrm{c}$ extends the data of Fig. $6 \mathrm{~b}$ by displaying the total MSHR occupancy for both load and store misses. The figure indicates that Radix has a large amount of store miss overlap. This overlap does not contribute to an increase in

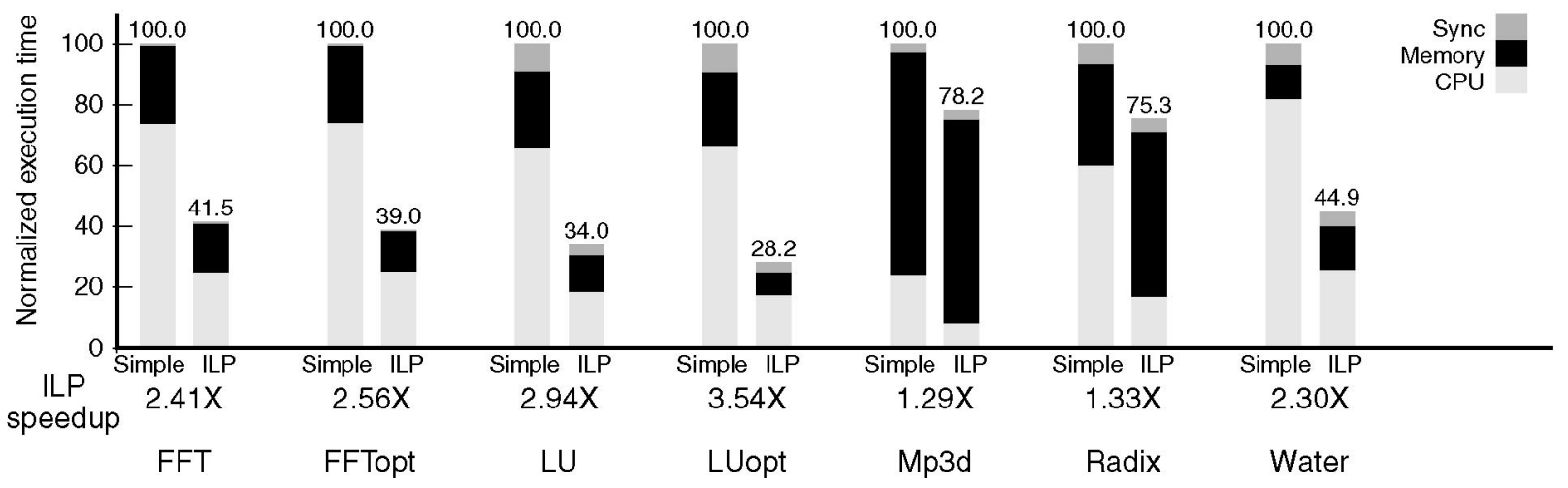

Fig. 3. Impact of ILP on multiprocessor performance. 


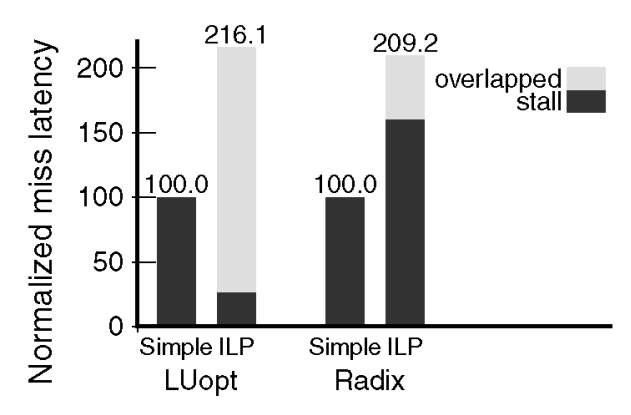

(a)

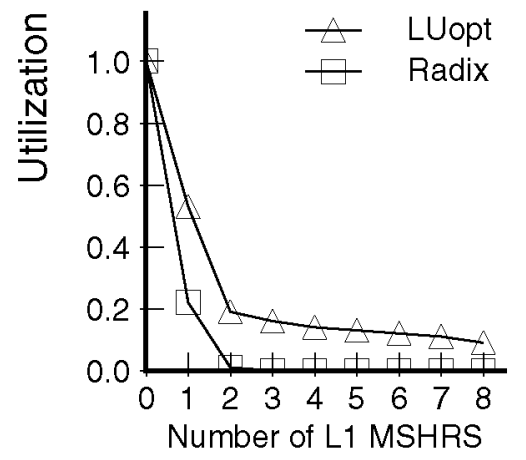

(b)

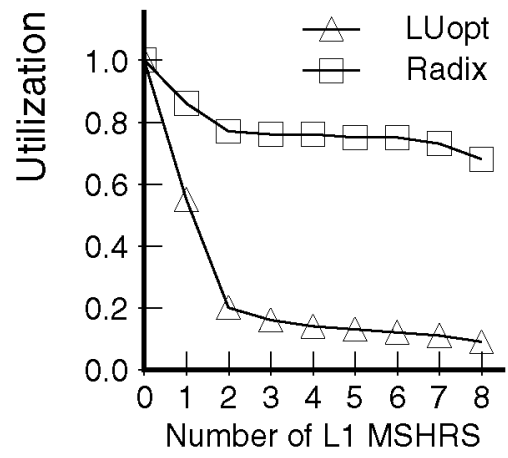

(c)

Fig. 6. Load miss overlap and contention in the ILP system.

memory ILP speedup since store latencies are already hidden in both the Simple and ILP systems due to release consistency. The store miss overlap, however, increases contention in the memory hierarchy, resulting in the ILP memory slowdown in Radix. In LUopt, the contentionrelated latency comes primarily from load misses, but its effect is mitigated since overlapped load misses contribute to reducing memory stall time.

\subsection{Memory Stall Component and Parallel Efficiency}

Using the above analysis, we can see why the ILP system generally sees a larger relative memory stall time component (Fig. 3) and a generally poorer parallel efficiency (Fig. 4) than the Simple system.

Since memory ILP speedup is generally less than CPU ILP speedup, the memory component becomes a greater fraction of total execution time in the ILP system than in the Simple system. To understand the reduced parallel efficiency, Fig. 7 provides the ILP speedups for the uniprocessor configuration for reference. The uniprocessor also generally sees lower memory ILP speedups than CPU ILP speedups. However, the impact of the lower memory ILP speedup is higher in the multiprocessor because the longer latencies of remote misses and increased contention result in a larger relative memory component in the execution time (relative to the uniprocessor). Additionally, the dichotomy between local and remote miss latencies in a multiprocessor often tends to decrease memory ILP speedup (relative to the uniprocessor), because load misses must

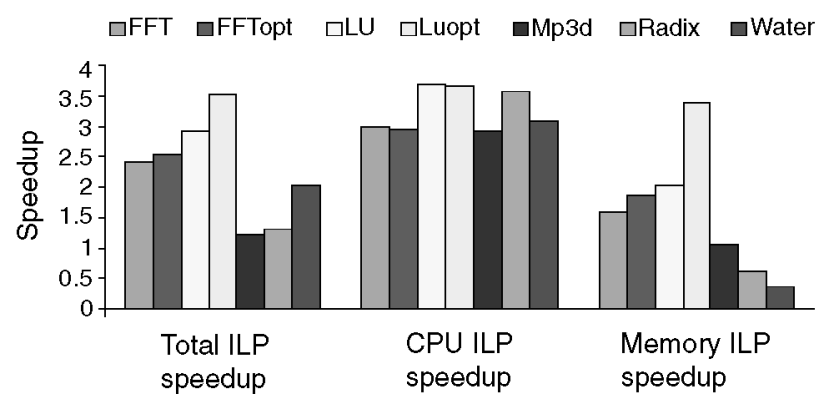

Fig. 5. ILP speedup for total execution time, CPU time, and memory stall time in the multiprocessor system. be overlapped not only with other load misses but with load misses with similar latencies. ${ }^{4}$ Thus, overall, the multiprocessor system is less able to exploit ILP features than the corresponding uniprocessor system for most applications. Consequently, the ILP multiprocessor generally sees lower parallel efficiency than the Simple multiprocessor.

\section{INTERACTION OF ILP TECHNIQUES WITH Software Prefetching}

The previous section shows that the ILP system sees a greater bottleneck from memory latency than the Simple system. Software-controlled nonbinding prefetching has been shown to effectively hide memory latency in sharedmemory multiprocessors with simple processors. This section evaluates how software prefetching interacts with ILP techniques in shared-memory multiprocessors. We followed the software prefetch algorithm developed by Mowry et al. [6] to insert prefetches in our applications by hand with one exception. The algorithm in [6] assumes that locality is not maintained across synchronization and, so, does not schedule prefetches across synchronization accesses. We removed this restriction when beneficial. For a consistent comparison, the experiments reported are with prefetches scheduled identically for both Simple and ILP; the prefetches are scheduled at least 200 dynamic instructions before their corresponding demand accesses. The impact of this scheduling decision is discussed below, including the impact of varying this prefetch distance.

\subsection{Overall Results}

Fig. 8 graphically presents the key results from our experiments (FFT and FFTopt have similar performance, so only FFTopt appears in the figure). The figure shows the execution time (and its components) for each application on Simple and ILP, both without and with software prefetching (+PF indicates the addition of software prefetching). Execution times are normalized to the time for the

4. FFT and FFTopt see better memory ILP speedups in the multiprocessor than in the uniprocessor because they overlap multiple load misses with similar multiprocessor (remote) latencies. The section of the code that exhibits overlap has a greater impact in the multiprocessor because of the longer remote latencies incurred in this section. 


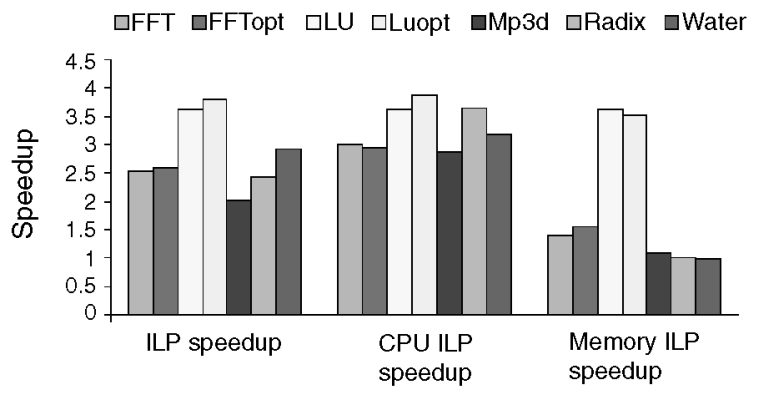

Fig. 7. ILP speedup for total execution time, CPU time, and memory stall time in the uniprocessor system.

application on Simple without prefetching. Fig. 9 summarizes some key data.

Software prefetching achieves significant reductions in execution time on ILP (13 percent to 43 percent) for three cases (LU, Mp3d, and Water). These reductions are similar to or greater than those in Simple for these applications. However, software prefetching is less effective at reducing memory stalls on ILP than on Simple (average reduction of 32 percent in ILP, ranging from 7 percent to 72 percent vs. average 59 percent and range of 21 percent to 88 percent in Simple). The net effect is that, even after prefetching is applied to ILP, the average memory stall time is 39 percent on ILP with a range of 11 percent to 65 percent (vs. average of 16 percent and range of 1 percent to 29 percent for Simple). For most applications, the ILP system remains largely memory-bound, even with software prefetching.

\subsection{Factors Contributing to the Effectiveness of Software Prefetching}

We next identify three factors that make software prefetching less successful in reducing memory stall time in ILP than in Simple, two factors that allow ILP additional benefits in memory stall reduction not available in Simple, and one factor that can either help or hurt ILP. We focus on issues that are specific to ILP systems; previous work has discussed non-ILP specific issues [6]. Fig. 10 summarizes the effects that were exhibited by the applications we studied. Of the negative effects, the first two are the most important for our applications.

\subsubsection{Increased Late Prefetches}

The last column of Fig. 9 shows that the number of prefetches that are too late to completely hide the miss latency increases in all our applications when moving from Simple to ILP. One reason for this increase is that multiple-issue and out-of-order scheduling speed up computation in ILP, decreasing the computation time with which each prefetch is overlapped. Simple also stalls on any load misses that are not prefetched or that incur a late prefetch, thereby allowing other outstanding prefetched data to arrive at the cache. ILP does not provide similar leeway.

\subsubsection{Increased Resource Contention}

As shown in Section 3, ILP processors stress system resources more than Simple. Prefetches further increase demand for resources, resulting in more contention and greater memory latencies. The resources most stressed in our configuration were cache ports, MSHRs, ALUs, and address generation units.

\subsubsection{Negative Interaction with Clustered Misses}

Optimizations to cluster load misses for the ILP system, as in LUopt, can potentially reduce the effectiveness of software prefetching. For example, the addition of prefetching reduces the execution time of LU by 13 percent on the ILP system; in contrast, LUopt improves by only 3 percent. (On the Simple system, both LU and LUopt improve by about 10 percent with prefetching.) LUopt with prefetching is slightly better than LU with prefetching on ILP (by 3 percent). The clustering optimization used in LUopt decreases the amount of computation between successive used in LUopt reduces the computation between successive misses, contributing to a high number of late prefetches and increased contention with prefetching.

\subsubsection{Overlapped Accesses}

In ILP, accesses that are difficult to prefetch may be overlapped because of nonblocking loads and out-of-order scheduling. Prefetched lines in LU and LUopt often suffer from L1 cache conflicts, resulting in these lines being replaced to the $\mathrm{L} 2$ cache before being used by the demand accesses. This L2 cache latency results in stall time in Simple, but can be overlapped by the processor in ILP. Since prefetching in ILP only needs to target those accesses that are not already overlapped by ILP, it can appear more effective in ILP than in Simple.

\subsubsection{Fewer Early Prefetches}

Early prefetches are those where the prefetched lines are either invalidated or replaced before their corresponding demand accesses. Early prefetches can hinder demand accesses by invalidating or replacing needed data from the same or other caches without providing any benefits in latency reduction. In many of our applications, the number of early prefetches drops in ILP, improving the effectiveness of prefetching for these applications. This reduction occurs because the ILP system allows less time between a prefetch and its subsequent demand access, decreasing the likelihood of an intervening invalidation or replacement.

\subsubsection{Speculative prefetches}

In ILP, prefetch instructions can be speculatively issued past a mispredicted branch. Speculative prefetches can potentially hurt performance by bringing unnecessary lines into the cache or by bringing needed lines into the cache too early. Speculative prefetches can also help performance by initiating a prefetch for a needed line early enough to hide its latency. In our applications, most prefetches issued past mispredicted branches were to lines also accessed on the correct path.

\subsection{Impact of Software Prefetching on Execution Time}

Despite its reduced effectiveness in addressing memory stall time, software prefetching achieves significant execution time reductions with ILP in three cases (LU, Mp3d, 


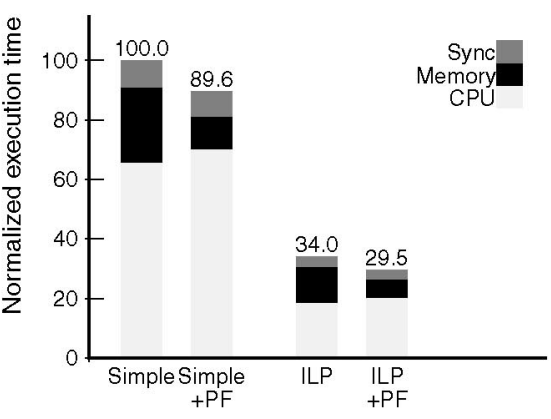

LU

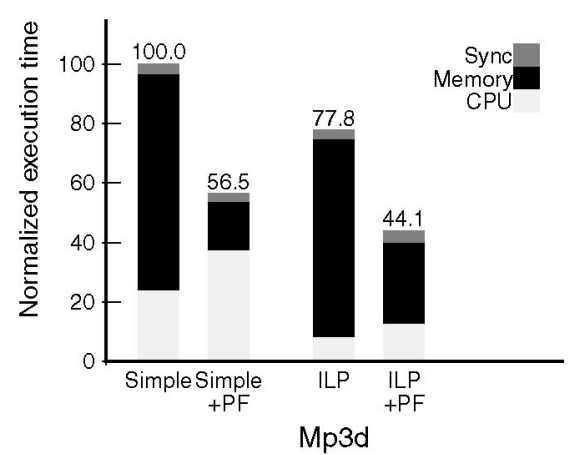

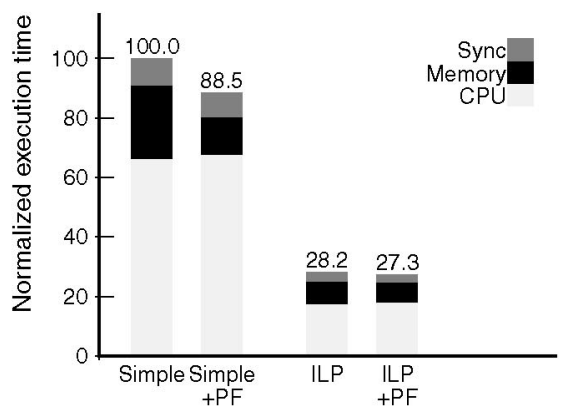

LUopt

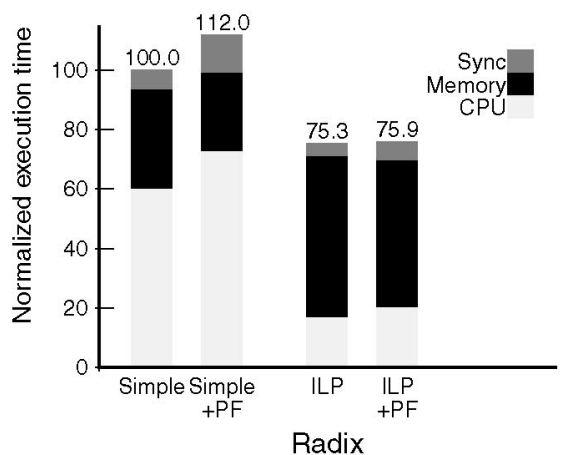

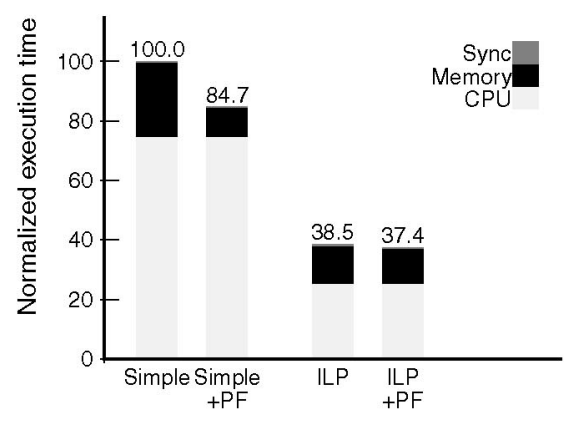

FFTopt

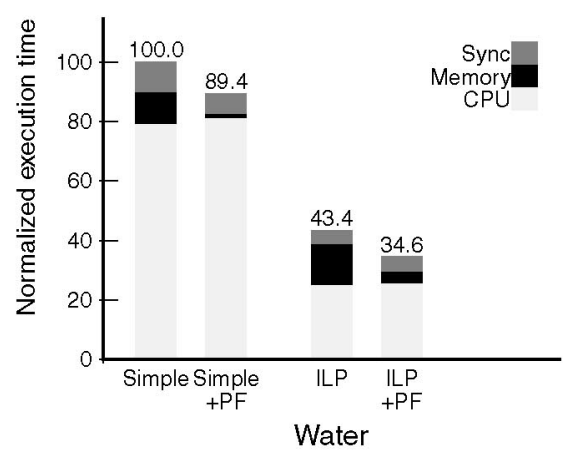

Fig. 8. Interaction between software prefetching and ILP.

\begin{tabular}{|c|c|c|c|c|c|c|c|c|}
\hline Application & $\begin{array}{l}\text { \% reductio } \\
\text { ecution tir }\end{array}$ & $\begin{array}{l}\text { i in ex- } \\
\text { e }\end{array}$ & $\begin{array}{l}\% \text { reduc } \\
\text { memory s }\end{array}$ & $\begin{array}{l}\text { on in } \\
\text { all time }\end{array}$ & $\begin{array}{l}\% \quad \text { re } \\
\text { memory s }\end{array}$ & $\begin{array}{l}\text { naining } \\
\text { all time }\end{array}$ & $\begin{array}{l}\% \text { prefetc } \\
\text { are late }\end{array}$ & es that \\
\hline & Simple & ILP & Simple & ILP & Simple & ILP & Simple & ILP \\
\hline $\mathrm{LU}$ & 10 & 13 & 57 & 49 & 12 & 21 & 4 & 40 \\
\hline LUopt & 11 & 3 & 49 & 14 & 14 & 24 & 12 & 35 \\
\hline FFTopt & 15 & 3 & 60 & 7 & 12 & 32 & 13 & 29 \\
\hline Mp3d & 43 & 43 & 78 & 59 & 29 & 62 & 1 & 12 \\
\hline Radix & -12 & -1 & 21 & 9 & 23 & 65 & 0 & 2 \\
\hline Water & 11 & 20 & 88 & 72 & 1 & 11 & 0 & 8 \\
\hline Average & 14 & 14 & 59 & 32 & 16 & 39 & 5 & 17 \\
\hline
\end{tabular}

Fig. 9. Detailed data on effectiveness of software prefetching. For the average, from LU and LUopt, only LUopt is considered since it provides better performance than LU with prefetching and ILP.

and Water) for two main reasons. First, memory stall time contributes a larger portion of total execution time in ILP. Thus, even a reduction of a small fraction of memory stall time can imply a reduction in overall execution time similar to or greater than that seen in Simple. Second, ILP systems see less instruction overhead from prefetching compared to Simple systems because ILP techniques allow the overlap of these instructions with other computation.

\subsection{Alleviating Late Prefetches and Contention}

Our results show that late prefetches and resource contention are the two key limitations to the effectiveness of prefetching on ILP. We tried several straightforward modifications to the prefetching algorithm and the system to address these limitations [12]. Specifically, we doubled and quadrupled the prefetch distance (i.e., the distance

\begin{tabular}{|l|c|c|c|c|c|c|}
\hline \multicolumn{1}{|c|}{ Factor } & LU & $\begin{array}{c}\text { LU } \\
\text { opt }\end{array}$ & $\begin{array}{c}\text { FFT } \\
\text { opt }\end{array}$ & Mp3d & Water & Radix \\
\hline \hline $\begin{array}{l}\text { Late } \\
\text { prefetches }\end{array}$ & $\sqrt{ }$ & $\sqrt{ }$ & $\sqrt{ }$ & $\sqrt{ }$ & $\sqrt{ }$ & $\sqrt{ }$ \\
\hline $\begin{array}{l}\text { Resource } \\
\text { contention }\end{array}$ & & $\sqrt{ }$ & $\sqrt{ }$ & & $\sqrt{ }$ & $\sqrt{ }$ \\
\hline $\begin{array}{l}\text { Clustered } \\
\text { load misses }\end{array}$ & & $\sqrt{ }$ & $\sqrt{ }$ & & & \\
\hline $\begin{array}{l}\text { Overlapped } \\
\text { accesses }\end{array}$ & $\sqrt{ }$ & $\sqrt{ }$ & & & & \\
\hline $\begin{array}{l}\text { Early } \\
\text { prefetches }\end{array}$ & $\sqrt{ }$ & $\sqrt{ }$ & & $\sqrt{ }$ & $\sqrt{ }$ & $\sqrt{ }$ \\
\hline $\begin{array}{l}\text { Speculative } \\
\text { prefetches }\end{array}$ & & & $\sqrt{ }$ & $\sqrt{ }$ & \\
\hline \hline
\end{tabular}

Fig. 10. Factors affecting the performance of prefetching for ILP. 
between a prefetch and the corresponding demand access) and increased the number of MSHRs. However, these modifications traded off benefits among late prefetches, early prefetches, and contention, without improving the combination of these factors enough to improve overall performance. We also tried varying the prefetch distance for each access according to the expected latency of that access (versus a common distance for all accesses) and prefetching only to the L2 cache. These modifications achieved their purpose, but did not provide a significant performance benefit for our applications [12].

\section{Discussion}

Our results show that shared-memory systems are limited in their effectiveness in exploiting ILP processors due to limited benefits of ILP techniques for the memory system. The analysis of Section 3 implies that the key reasons for the limited benefits are the lack of opportunity for overlapping load misses and/or increased contention in the system. Compiler optimizations akin to the loop interchanges used to generate LUopt and FFTopt may be able to expose more potential for load miss overlap in an application. The simple loop interchange used in LUopt provides a 13 percent reduction in execution time compared to LU on an ILP multiprocessor. Hardware enhancements can also increase load miss overlap, e.g., through a larger instruction window. Targeting contention requires increased hardware resources, or other latency reduction techniques.

The results of Section 4 show that, while software prefetching improves memory system performance with ILP processors, it does not change the memory-bound nature of these systems for most of the applications because the latencies are too long to hide with prefetching and/or because of increased contention. Our results motivate prefetching algorithms that are sensitive to increases in resource usage. They also motivate latency-reducing (rather than tolerating) techniques, such as producer-initiated communication, which can improve the effectiveness of prefetching [1].

\section{Conclusions}

This paper evaluates the impact of ILP techniques supported by state-of-the-art processors on the performance of shared-memory multiprocessors. All our applications see performance improvements from current ILP techniques. However, while ILP techniques effectively address the CPU component of execution time, they are less successful in improving data memory stall time. These applications do not see the full benefit of the latency-tolerating features of ILP processors because of insufficient opportunities to overlap multiple load misses and increased contention for system resources from more frequent memory accesses. Thus, ILP-based multiprocessors see a larger bottleneck from memory system performance and generally poorer parallel efficiencies than previous-generation multiprocessors.

Software-controlled nonbinding prefetching is a latency hiding technique widely recommended for previous-generation shared-memory multiprocessors. We find that, while software prefetching results in substantial reductions in execution time for some cases on the ILP system, increased late prefetches and increased contention for resources cause software prefetching to be less effective in reducing memory stall time in ILP-based systems. Even after the addition of software prefetching, most of our applications remain largely memory bound.

Thus, despite the latency-tolerating techniques integrated within ILP processors, multiprocessors built from ILP processors have a greater need for additional techniques to hide or reduce memory latency than previousgeneration multiprocessors. One ILP-specific technique discussed in this paper is the software clustering of load misses. Additionally, latency-reducing techniques, such as producer-initiated communication, that can improve the effectiveness of prefetching appear promising.

\section{ACKNOWLEDGMENTS}

This work is supported in part by an IBM Partnership Award, Intel Corp., the U.S. National Science Foundation under Grant No. CCR-9410457, CCR-9502500, CDA9502791, and CDA-9617383, and the Texas Advanced Technology Program under Grant No. 003604-025. Sarita Adve is also supported by an Alfred P. Sloan Research Fellowship, Vijay S. Pai by a Fannie and John Hertz Foundation Fellowship, and Parthasarathy Ranganathan by a Lodieska Stockbridge Vaughan Fellowship.

\section{REFERENCES}

[1] H. Abdel-Shafi et al. , "An Evaluation of Fine-Grain Producer-Initiated Communication in Cache-Coherent Multiprocessors," Proc. Third Int'l Symp. High-Performance Computer Architecture, pp. 204-215, 1997.

[2] E.H. Gornish, "Adaptive and Integrated Data Cache Prefetching for Shared-Memory Multiprocessors," PhD thesis, Univ. of Illinois at Urbana-Champaign, 1995.

[3] D. Kroft, "Lockup-Free Instruction Fetch/Prefetch Cache Organization," Proc. Eighth Int'l Symp. Computer Architecture, pp. 81-87, 1981

[4] J. Laudon and D. Lenoski, "The SGI Origin 2000: A ccNUMA Highly Scalable Server," Proc. 24th Int'l Symp. Computer Architecture, pp. 241-251, 1997.

[5] C.-K. Luk and T.C. Mowry, "Compiler-Based Prefetching for Recursive Data Structures," Proc. Seventh Int'l Conf. Architectural Support for Programming Languages and Operating Systems, pp. 222234, 1996.

[6] T. Mowry, "Tolerating Latency through Software-Controlled Data Prefetching," PhD thesis, Stanford Univ., 1994.

[7] B.A. Nayfeh et al. , "Evaluation of Design Alternatives for a Multiprocessor Microprocessor," Proc. 23rd Int'l Symp. Computer Architecture, pp. 67-77, 1996.

[8] K. Olukotun et al. , "The Case for a Single-Chip Multiprocessor," Proc. Seventh Int'l Conf. Architectural Support for Programming Languages and Operating Systems, pp. 2-11, 1996.

[9] V.S. Pai et al. , "An Evaluation of Memory Consistency Models for Shared-Memory Systems with ILP Processors," Proc. Seventh Int'l Conf. Architectural Support for Programming Languages and Operating Systems, pp. 72-83, 1996.

[10] V.S. Pai et al., RSIM Reference Manual, Version 1.0, ECE TR 9705, Rice Univ., 1997

[11] V.S. Pai et al. , "The Impact of Instruction Level Parallelism on Multiprocessor Performance and Simulation Methodology," Proc. Third Int'l Symp. High Performance Computer Architecture, pp. 72-83, 1997.

[12] P. Ranganathan et al. , "The Interaction of Software Prefetching with ILP Processors in Shared-Memory Systems," Proc. 24th Int'l Symp. Computer Architecture, pp. 144-156, 1997. 
[13] J.P. Singh et al. , "SPLASH: Stanford Parallel Applications for Shared-Memory," Computer Architecture News, pp. 5-44, Mar. 1992.

[14] S.C. Woo et al. , "The SPLASH-2 Programs: Characterization and Methodological Considerations," Proc. 22nd Int'l Symp. Computer Architecture, pp. 24-36, 1995.

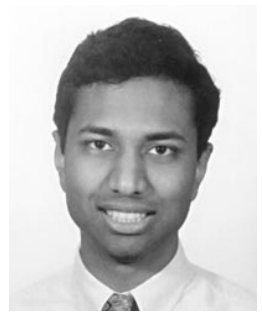

Vijay S. Pai received a BSEE degree in 1994 and an MS degree in electrical engineering in 1997, both from Rice University. He is currently a $\mathrm{PhD}$ candidate in the Department of Electrical and Computer Engineering at Rice University. His graduate education has been partly supported by a Fannie and John Hertz Foundation graduate fellowship. His research interests include uniprocessor and multiprocessor computer architecture, compilation, and performance evaluation, with a focus on effectively exploiting instructionlevel parallelism in multiprocessors. He is also a primary developer and maintainer of the publicly-available Rice Simulator for ILP Multiprocessors (RSIM). He is a student member of the ACM and the IEEE Computer Society and a member of the Eta Kappa Nu, Phi Beta Kappa, and Tau Beta Pi honorary societies.

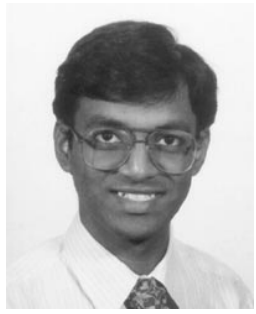

Parthasarathy Ranganathan received his BTech degree from the Indian Institute of Technology, Madras, in 1994 and his MS degree from Rice University in 1997 . He is currently a doctoral candidate in the Department of Electrical and Computer Engineering at Rice University. His broad research areas are in highperformance computer architecture and performance evaluation. He is a primary developer and maintainer of the publicly distributed Rice Simulator for ILP Multiprocessors (RSIM) infrastructure. He is currently working on developing cost-effective high-performance uniprocessor and multiprocessor systems for commercial database and multimedia applications. He is a student member of the ACM and the IEEE Computer Society, a member of Eta Kappa $\mathrm{Nu}$, and a recipient of the Lodieska Stockbridge Vaughan fellowship.

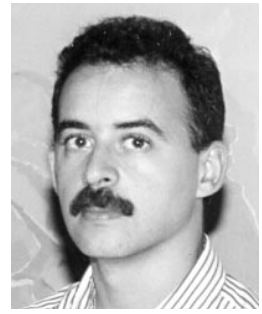

Hazim Abdel-Shafi received his BSEE from Santa Clara University and his MS in electrical engineering from Rice University. He is currently a doctoral student in the Electrical and Computer Engineering Department of Rice University. His main area of research is parallel and distributed processing. Specific areas of interest include techniques to tolerate and reduce memory latency in shared-memory multiprocessors, architectural support for multimedia applications, and reliability and computation migration in distributed computing environments. He is a student member of the ACM and the IEEE Computer Society, and a member of Alpha Sigma Nu and Tau Beta Pi.

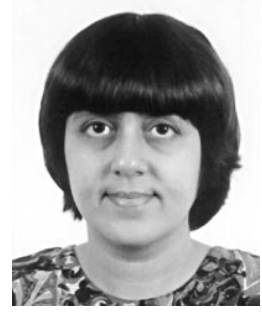

Sarita Adve received a BTech degree in electrical engineering from the Indian Institute of Technology-Bombay in 1987, and the MS and $\mathrm{PhD}$ degrees in computer science from the University of Wisconsin-Madison in 1989 and 1993, respectively. She is currently an assistant professor in the Department of Electrical and Computer Engineering at Rice University. Her research interests are in computer architecture, parallel computing, and performance evaluation methods. She received a U.S. National Science Foundation CAREER award in 1995, an IBM University Partnership award in 1997 and 1998, and an Alfred P. Sloan Research Fellowship in 1998. She is an associate editor for the ACM Transactions on Modeling and Computer Simulation and has served on several conference program committees. She is a member of the ACM and the IEEE Computer Society. 